\title{
Robert M. Carey, Hypertension and Hormone Mechanisms
}

\author{
Humana Press, Totowa, NJ, 2007, 378 pp, with illus, \$149, ISBN 9781588294081
}

\author{
David A. Calhoun
}

Received: 30 January 2008 / Accepted: 30 January 2008/Published online: 23 May 2008

(C) Humana Press Inc. 2008

A growing number of studies link various hormones, either when deficient or in excess, to the development of hypertension and vascular abnormalities. Historically, this has been most true of angiotensin II and aldosterone, but similar effects are being increasingly attributed to other less classical hormones such as endothelin, angiotensin (1-7), calcitonin, dopamine, free fatty acids, nitric oxide, kallikrein, natriuretic peptides, sex steroids and products of lipoxygenase enzymes. Given this rapidly expanding literature, this book provides a very timely overview of current results delineating the role that these and other hormones play in contributing to hypertension and its sequela. As edited by Dr. Carey, a well-recognized investigator in this field, leading experts provide very comprehensive discussions of the effect of the different hormonal systems on progression of cardiovascular disease. Overall, there is an emphasis on the vascular and cellular effects of the different hormones, but also included is relevant consideration of data linking hormones to true clinical concerns including hypertension, metabolic syndrome and obesity. In this regard, a very interesting chapter on the historical evolution of guidelines for diagnosing and treating hypertension is provided by Henry Black and colleagues. James Sowers contributes a very effective clinical review of the metabolic syndrome, including practical recommendations regarding use of different classes of antihypertensive agents in patients with the metabolic syndrome. Accordingly, this book provides a nice combination of both basic science and clinical considerations of hormonal causes of hypertension and vascular disease. What it does not provide is recommendations for evaluating and treating classical hypertensive syndromes of hormonal excess such as primary aldosteronism, Cushing's or pheochromocytoma. Overall, the book will serve as a valuable resource for scientists and clinicians interested in the underlying mechanisms of hypertension and vascular disease.

D. A. Calhoun $(\bowtie)$

University of Alabama at Birmingham, Birmingham, Alabama,

USA

e-mail: dcalhoun@uab.edu 